\title{
Directional Sub-THz Antenna-Channel Modelling for Indoor Scenarios
}

\author{
Brecht De Beelde, David Plets, Emmeric Tanghe, Wout Joseph \\ Ghent University - IMEC, Ghent, Belgium, Brecht.DeBeelde@UGent.be
}

\begin{abstract}
In this paper, sub-THz channel sounding is discussed for indoor scenarios. A directional D-band channel sounder, operational in the full band ranging from 110 to $170 \mathrm{GHz}$, is presented. We examine the influence of the antenna characteristics on the channel sounder. The channel sounder is used for Line-of-Sight path loss (PL) modelling at sub-THz frequencies for distances up to $4 \mathrm{~m}$. Validation measurements confirm the small beamwidth of the antenna, ranging from 12 degrees for $110 \mathrm{GHz}$ to 11 degrees for $170 \mathrm{GHz}$. An antenna de-embedding methodology is presented. Fitting Line-of-Sight $P L$ at center frequency $140 \mathrm{GHz}$ to a one-slope model gives a reference PL of 76.0 dB at $1 \mathrm{~m}$ and a PL exponent of 1.9. The reference $P L$ is slightly higher than free space $P L$, whereas the PL exponent is slightly lower.
\end{abstract}

Index Terms-sub-THz, D-band, channel characterization, indoor, path loss, modelling, channel sounding, antenna, beamwidth

\section{INTRODUCTION}

Whereas the radio channel below $100 \mathrm{GHz}$ is already well investigated for typical indoor scenarios [1]-[3], little is known about the radio channel above $100 \mathrm{GHz}$. In the Dband, ranging from 110 to $170 \mathrm{GHz}$, the $60 \mathrm{GHz}$ available bandwidth suffers less than the $60 \mathrm{GHz}$ mmWave frequency band from atmospheric absorption [4]. The D-band could be used for applications requiring ultra-high bandwidth [5] such as data kiosks [6], fixed wireless access [7] and virtual reality [8].

Radio propagation in a shopping mall at $140 \mathrm{GHz}$ is presented in [9] for distances up to $65 \mathrm{~m}$, using a vector network analyzer (VNA) based channel sounder with a bandwidth of $4 \mathrm{GHz}$. The authors of [4] present a sliding correlation channel sounder which has an equal bandwidth of $4 \mathrm{GHz}$. This channel sounder is used for modelling indoor Line-of-Sight (LOS) and non-LOS (NLOS) path loss (PL) for distances up to $40 \mathrm{~m}$ [10], reporting a PL exponent of 2.0 for LOS measurements and up to 4.7 for NLOS measurements. LOS and non-LOS measurements in a subset of the D-band, i.e. 126 to $156 \mathrm{GHz}$, are performed in [11] and [12]. [12] reports PL exponents as low as 1.45 for indoor LOS scenarios. The full D-band is considered in [13], using a VNA-based approach to compare PL models for indoor channels with distances up to $86 \mathrm{~cm}$ and emphasizing the need for proper antenna de-embedding. Indoor D-band channel measurements are also presented in [14], considering LOS PL as well as channel properties

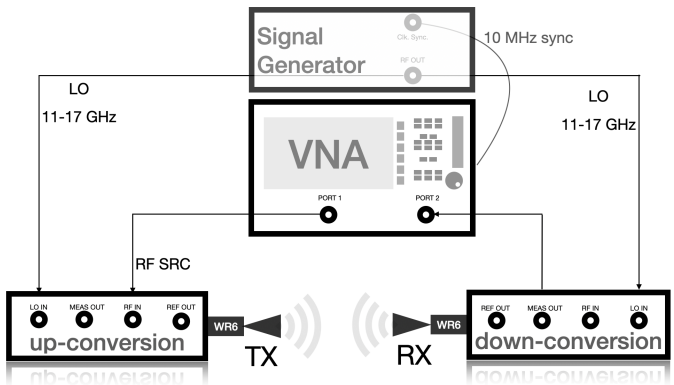

Fig. 1: Channel sounder overview

of obstructed and NLOS environments. Similar to the PL models in [13], the distance in [14] is limited to less than $1 \mathrm{~m}$.

The goal of this paper is to assess the antenna influence on the channel sounder setup and perform indoor LOS PL measurements with distances up to $4 \mathrm{~m}$, considering the full D-band ranging from $110 \mathrm{GHz}$ to $170 \mathrm{GHz}$. To the best of the authors' knowledge, this is the first time that the full D-band is characterized for distances larger than $1 \mathrm{~m}$. The antenna de-embedding methodology will be applied for other planned indoor measurement campaigns.

\section{Methodology}

In this section, we present the methodology for the antenna-channel measurements. We start with the channel sounder design in Sect. II-A. In Sect. II-B we present the measurements we performed to confirm the antenna gain of antennas we used in our channel sounder. The methodology for validating the antennas' $-3 \mathrm{~dB}$ beamwidth is outlined in Sect. II-C. After validation of the channel sounder and antenna model, we use the sounder setup for D-band LOS PL modelling in a laboratory setting in Sect. II-D.

\section{A. D-band channel sounder}

We use a VNA-based channel sounding approach using a two-port VNA operational up to $67 \mathrm{GHz}$. The overall setup of our channel sounder is shown in Fig. 1. The RF input source, generated at port 1 of the VNA, is up-converted via a R\&S ZC170 mixer using a local oscillator (LO) signal generated by an external signal generator. 


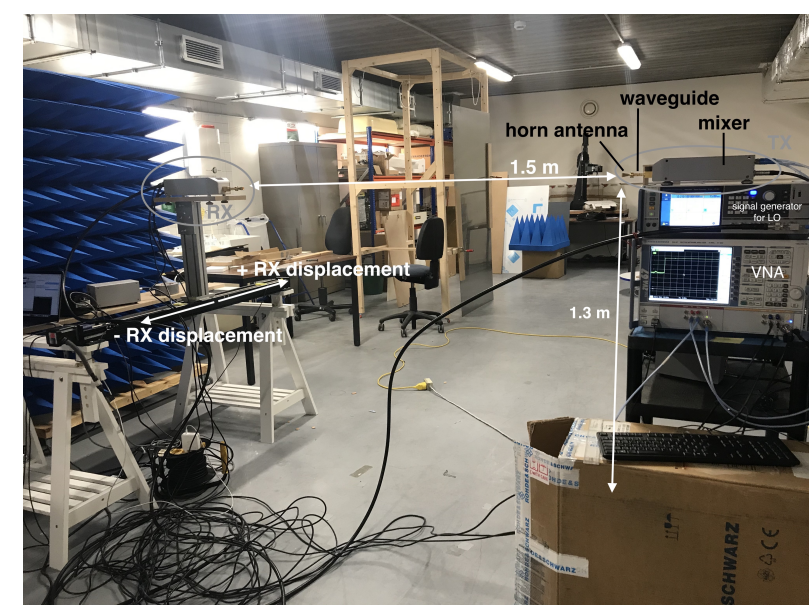

Fig. 2: Validation measurement setup

A QuinStar pyramidal standard horn antenna, connected to the mixer's WR-6 waveguide, transmits the signal. An identical horn antenna at the receiver side captures the Dband signal which is down-converted via a second $\mathrm{ZC} 170$ mixer using the same LO signal and sent to port 2 of the VNA. We get the channel transfer function by measuring the $S_{21}$ scattering parameter. The horn antennas have a reported gain of $22.2 \mathrm{dBi}$ for $110 \mathrm{GHz}$ up to $23.2 \mathrm{dBi}$ for $170 \mathrm{GHz}$ [15]. The H-plane half-power beamwidth (HPBW) ranges from 12.5 degrees for $110 \mathrm{GHz}$ to 12.0 degrees for $170 \mathrm{GHz}$.

With this setup, we perform a frequency sweep that covers the full D-band. Over this $60 \mathrm{GHz}$ band we take 3001 frequency points with a $20 \mathrm{MHz}$ interspacing and an IF measurement bandwidth of $100 \mathrm{~Hz}$. Before starting the measurements, a normalized through calibration is performed to account for cable and connector losses and phase differences. The signal to noise ratio (SNR) of the VNA is $-75 \mathrm{~dB}$, which results in a dynamic range of the sounder of $120 \mathrm{~dB}$, taking into account the antenna gains of $22.5 \mathrm{dBi}$.

The Fraunhofer far field distance $d_{F}$ of the horn antennas, with $D$ the diameter of the horn aperture and $\lambda$ the wavelength at frequency $170 \mathrm{GHz}$, is $0.55 \mathrm{~m}$.

$$
d_{F}=\frac{2 D^{2}}{\lambda}
$$

\section{B. Channel sounder validation}

A picture of the channel sounder setup for the validation measurements is shown in Fig. 2. For the validation of the channel sounder, we performed 6 Line-of-Sight (LOS) measurements on two different days with a distance of $1.5 \mathrm{~m}$ between the transmit (TX) and receive (RX) antennas. Both antennas are levelled and placed at a height of $1.3 \mathrm{~m}$ above the ground and $1.3 \mathrm{~m}$ below the ceiling which, due to the narrow antenna beamwidth, ensures that no reflections on ground or ceiling are received. We obtain measured system loss (SL) as a function of frequency $f$ via (2), with $H$ the measured transfer function. This SL contains path loss (PL) and antenna gains.

$$
\operatorname{SL}_{\mathrm{dB}}(f)=-10 \log _{10}\left(|H(f)|^{2}\right)
$$

We then calculate free space system loss (FSSL) via (3), with $G_{a}$ the frequency-dependent antenna gain obtained from [15], $d$ the distance in meter and $c$ the speed of light.

$$
\operatorname{FSSL}_{\mathrm{dB}}(f)=20 \log _{10}\left(4 \pi d \frac{f}{c}\right)-2 G_{a}(f)
$$

We expect the measured SL to match the calculated FSSL. The difference between FSSL and SL is stored as correction data.

\section{Antenna beamwidth validation}

As can be seen in the picture from Fig. 2, the RX antenna is placed on a Velmex BiSlide positioning system with sub-millimeter precision, which we use to change the RX location from $-45 \mathrm{~cm}$ to $+45 \mathrm{~cm}$ compared to the laser aligned reference location, in steps of $1 \mathrm{~cm}$. For every RX displacement, we add the correction data to the measured SL and use the actual distance between the two antennas to calculate the free-space path loss (FSPL) for that specific displacement. The antenna gain for a given azimuth is then obtained by adding the FSPL to the corrected measured SL. We then divide the result by two, as two identical antennas are used and due to the geometry of the setup. Finally, we transform the RX displacement to angles in the azimuth domain via simple geometry and create a polar plot of the measured antenna gain, averaged in the frequency band from 120 to $130 \mathrm{GHz}$.

\section{Path loss model}

After the validation, the channel sounder is used to model LOS PL in a laboratory setting. Compared to the validation measurements, which were performed in the center of the lab without any nearby reflectors, the PL measurements were performed closer to the wall and with measurement equipment nearby. In the measurement setup, the RX antenna is fixed, while the TX antenna is pulled away from the RX antenna. The distance between the two antennas ranges from $20 \mathrm{~cm}$ to $4 \mathrm{~m}$, in steps of $10 \mathrm{~cm}$. At every location, two frequency sweeps are performed and averaged. We de-embed the TX and RX antennas by adding the antenna gains $G_{a}$ and adding the correction data from Sect. II-B.

We fit the PL as a function of distance to the linear model from (4), with $d$ the distance in meter between the antennas, $P L_{0}$ the reference PL in $\mathrm{dB}$ at $1 \mathrm{~m}, n$ the PL exponent and $\chi$ the shadow fading term based on a zero-mean normal distribution with standard deviation $\sigma$.

$$
\mathrm{PL}_{d B}(d)=P L_{0}+10 n \log _{10}(d / 1 \mathrm{~m})+\chi
$$




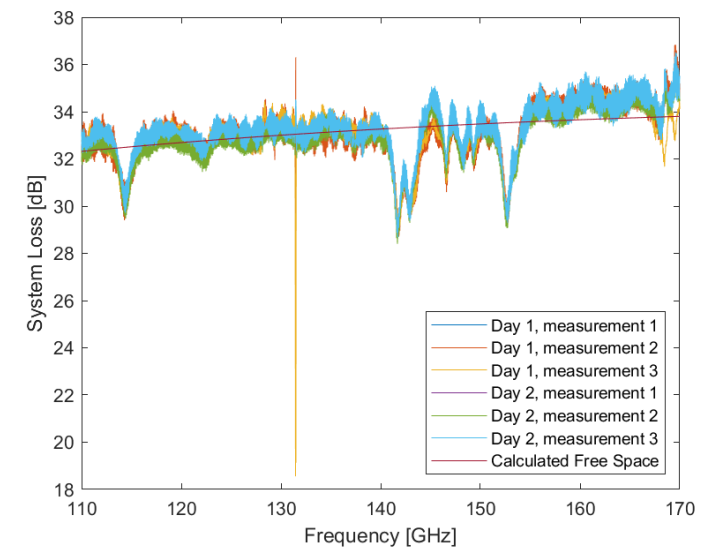

Fig. 3: Reference measurement with $d=1.5 \mathrm{~m}$ and both antennas perfectly aligned

\section{RESULTS}

\section{A. Reference measurements}

The measured SL from (2) is shown in Fig. 3 together with the calculated FSSL from (3). The measurements are stable and reproducible, with a maximum difference of $1 \mathrm{~dB}$ between different measurements. Overall, the measured SL corresponds to the calculated FSSL, except for some underestimations of 2 to $4 \mathrm{~dB}$ at frequencies 112, 142 and $152 \mathrm{GHz}$. As we performed a normalized through calibration with the WR-6 waveguides as reference plane and as we see these peaks in all our measurements, we expect that these underestimations are caused by an increased antenna gain at these frequencies. The averaged difference between measured SL and calculated FSSL is stored as correction data.

\section{B. Antenna beamwidth validation}

The measured antenna gain as a function of RX displacement is shown in Fig. 4a. After transformation from RX displacement to azimuth angles we obtain the polar plot of Fig. 4b. At $140 \mathrm{GHz}$, the $-3 \mathrm{~dB}$ beamwidth of the antenna is $11.4^{\circ}$, which is $1^{\circ}$ lower than the horn's reported Hplane beamwidth. As expected, the beamwidth decreases with frequency, ranging from $12.2^{\circ}$ at $110 \mathrm{GHz}$ to $11.2^{\circ}$ at $170 \mathrm{GHz}$.

\section{Line-of-Sight path loss model}

Figure 5a shows the corrected PL as a function of frequency for distances $0.5,2$ and $4 \mathrm{~m}$, together with the free space PL for these distances. The measured PL, after antenna de-embedding, is just slightly higher than the FSPL. The measured PL as a function of distance is shown in Fig. $5 b$ for the frequencies 110,140 and $170 \mathrm{GHz}$, together with the free space PL for these frequencies. The fitted oneslope PL model from (4) for these frequencies is listed in

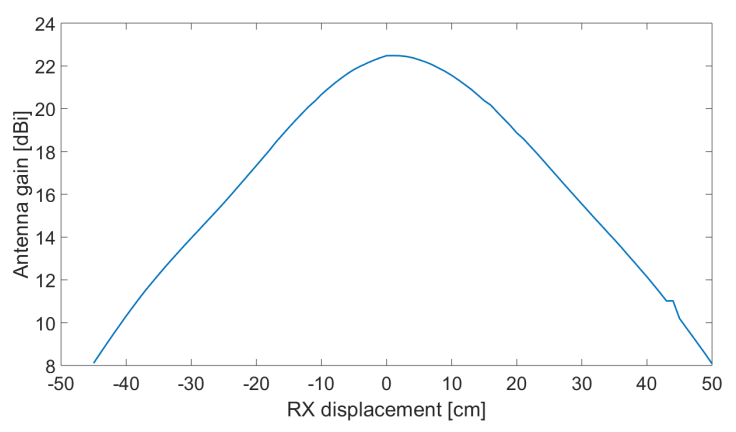

(a) Measured antenna gain as a function of RX displacement

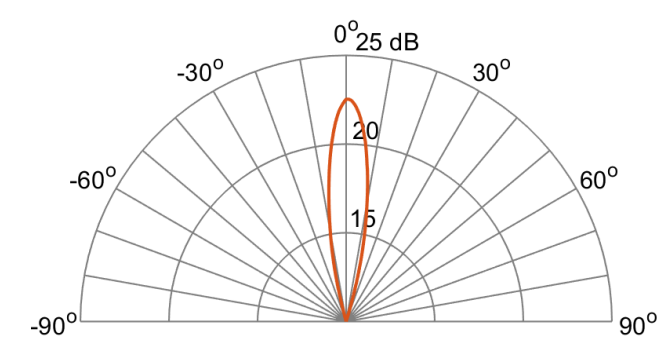

(b) Measured radiation pattern

Fig. 4: Antenna beamwidth validation

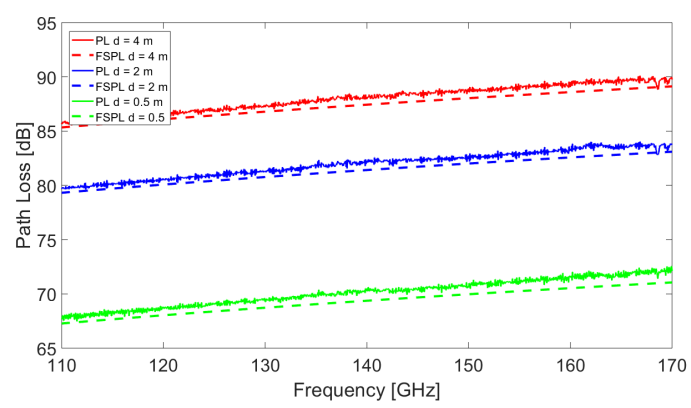

(a) Path loss as a function of frequency

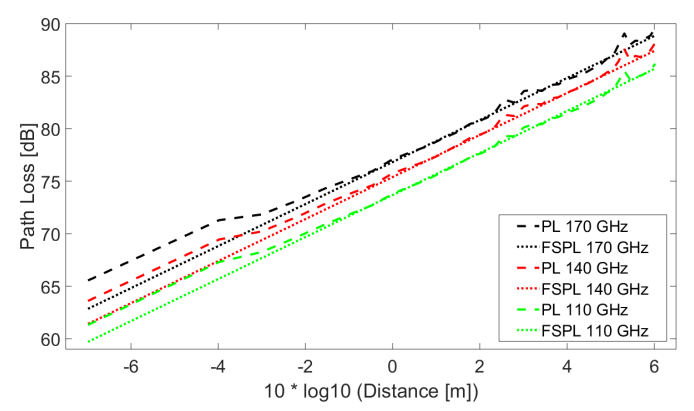

(b) Path loss as a function of distance

Fig. 5: Measured and free space path loss 
TABLE I: Fitted one-slope PL model

\begin{tabular}{|l|c|c|c|c|c|}
\hline Frequency & $P L_{0}[\mathrm{~dB}]$ & $n$ & $\sigma / \chi$ & $R^{2}$ & FSPL at $1 \mathrm{~m}$ \\
\hline $110 \mathrm{GHz}$ & 73.7 & 1.9 & $0.36 / 0.7 \mathrm{~dB}$ & 0.996 & $73.3 \mathrm{~dB}$ \\
$140 \mathrm{GHz}$ & 76.0 & 1.9 & $0.44 / 0.9 \mathrm{~dB}$ & 0.995 & $75.4 \mathrm{~dB}$ \\
$170 \mathrm{GHz}$ & 77.5 & 1.8 & $0.60 / 1.2 \mathrm{~dB}$ & 0.989 & $77.1 \mathrm{~dB}$ \\
\hline
\end{tabular}

Table I, together with a shadow fading term $\chi$ corresponding to the $95 \%$ percentile. The PL exponent $n$ is almost 2 , which was to be expected as no obstructions were present in the LOS path. This is also in line with the results presented in [13] and [14], but lower than the fitted PL model of [4], where a PL exponent of 2.02 is reported.

\section{CONCLUSION}

In this paper, we presented a channel sounder for Dband channel measurements. The channel sounder setup has been validated and the beamwidth measurements show a $-3 \mathrm{~dB}$ beamwidth of $11.4^{\circ}$, which is slightly smaller than the reported beamwidth of $12^{\circ}$. The channel sounder is used for directional Line-of-Sight path loss modelling in a laboratory environment, which results in a reference path loss that is slightly higher than the corresponding free space path loss and a path loss exponent of 1.9 which is slightly lower than free space.

\section{ACKNOWLEDGMENT}

This work was executed within the imec AAA D-band channel modelling research project D-BARC and EOS project MULti-Service WIreless NETwork MUSE-WINET. D-BARC received support from Flanders Innovation \& Entrepreneurship. The authors would like to thank André Bourdoux, Claude Desset and Joris Van Driessche for their input in the sounder design.

\section{REFERENCES}

[1] H. Hashemi, "The indoor radio propagation channel," Proceedings of the IEEE, vol. 81, no. 7, pp. 943-968, 1993.

[2] T. K. Sarkar, Zhong Ji, Kyungjung Kim, A. Medouri, and M. SalazarPalma, "A survey of various propagation models for mobile communication," IEEE Antennas and Propagation Magazine, vol. 45, no. 3 , pp. 51-82, 2003.
[3] H. Obeidat, A. Alabdullah, E. Elkhazmi, W. Suhaib, O. Obeidat, M. Alkhambashi, M. Mosleh, N. Ali, Y. Dama, Z. Abidin, R. AbdAlhameed, and P. Excell, "Indoor environment propagation review," Computer Science Review, vol. 37, p. 100 272, 2020.

[4] Y. Xing and T. S. Rappaport, "Propagation measurement system and approach at $140 \mathrm{ghz}$-moving to $6 \mathrm{~g}$ and above $100 \mathrm{ghz}$," in 2018 IEEE Global Communications Conference (GLOBECOM), 2018, pp. 1-6.

[5] H. Sarieddeen, N. Saeed, T. Y. Al-Naffouri, and M. Alouini, "Next generation terahertz communications: A rendezvous of sensing, imaging, and localization," IEEE Commun. Mag., vol. 58, no. 5, pp. 69-75, 2020.

[6] S. Priebe, C. Jastrow, M. Jacob, T. Kleine-Ostmann, T. Schrader, and T. Kürner, "Channel and propagation measurements at 300 ghz," IEEE Transactions on Antennas and Propagation, vol. 59, no. 5, pp. 1688-1698, 2011.

[7] I. F. Akyildiz, A. Kak, and S. Nie, "6g and beyond: The future of wireless communications systems," IEEE Access, vol. 8, pp. 133 995-134 030, 2020.

[8] Y. Khorsandmanesh and M. J. Emadi, "Peak age of information analysis for virtual reality in terahertz communications," in 2020 Iran Workshop on Communication and Information Theory (IWCIT), 2020, pp. $1-6$.

[9] S. L. H. Nguyen, J. Järveläinen, A. Karttunen, K. Haneda, and J. Putkonen, "Comparing radio propagation channels between 28 and $140 \mathrm{ghz}$ bands in a shopping mall," in 12th European Conference on Antennas and Propagation (EuCAP 2018), 2018, pp. 1-5.

[10] Y. Xing, O. Kanhere, S. Ju, and T. Rappaport, "Indoor wireless channel properties at millimeter wave and sub-terahertz frequencies," 12 2019, pp. 1-6.

[11] L. Pometcu and R. D'Errico, “Channel model characteristics in dband for nlos indoor scenarios," in 2019 13th European Conference on Antennas and Propagation (EuCAP), 2019, pp. 1-4.

[12] L. Pometcu and R. D'Errico, "An indoor channel model for high datarate communications in d-band," IEEE Access, vol. 8, pp. 9420-9433, 2020.

[13] C. Cheng, S. Kim, and A. Zajić, "Comparison of path loss models for indoor $30 \mathrm{ghz}, 140 \mathrm{ghz}$, and $300 \mathrm{ghz}$ channels," in 2017 11th European Conference on Antennas and Propagation (EUCAP), 2017, pp. 716-720.

[14] S. Kim, W. T. Khan, A. Zajić, and J. Papapolymerou, "D-band channel measurements and characterization for indoor applications," IEEE Transactions on Antennas and Propagation, vol. 63, no. 7, pp. 3198-3207, 2015.

[15] Standard Gain Horn Antennas - QWH-DPRR0O, QuinStar Technology, Inc, May 2020. 